\title{
Il mercato dei dispositivi per stomia in cinque paesi europei: lezioni per l'Italia
}

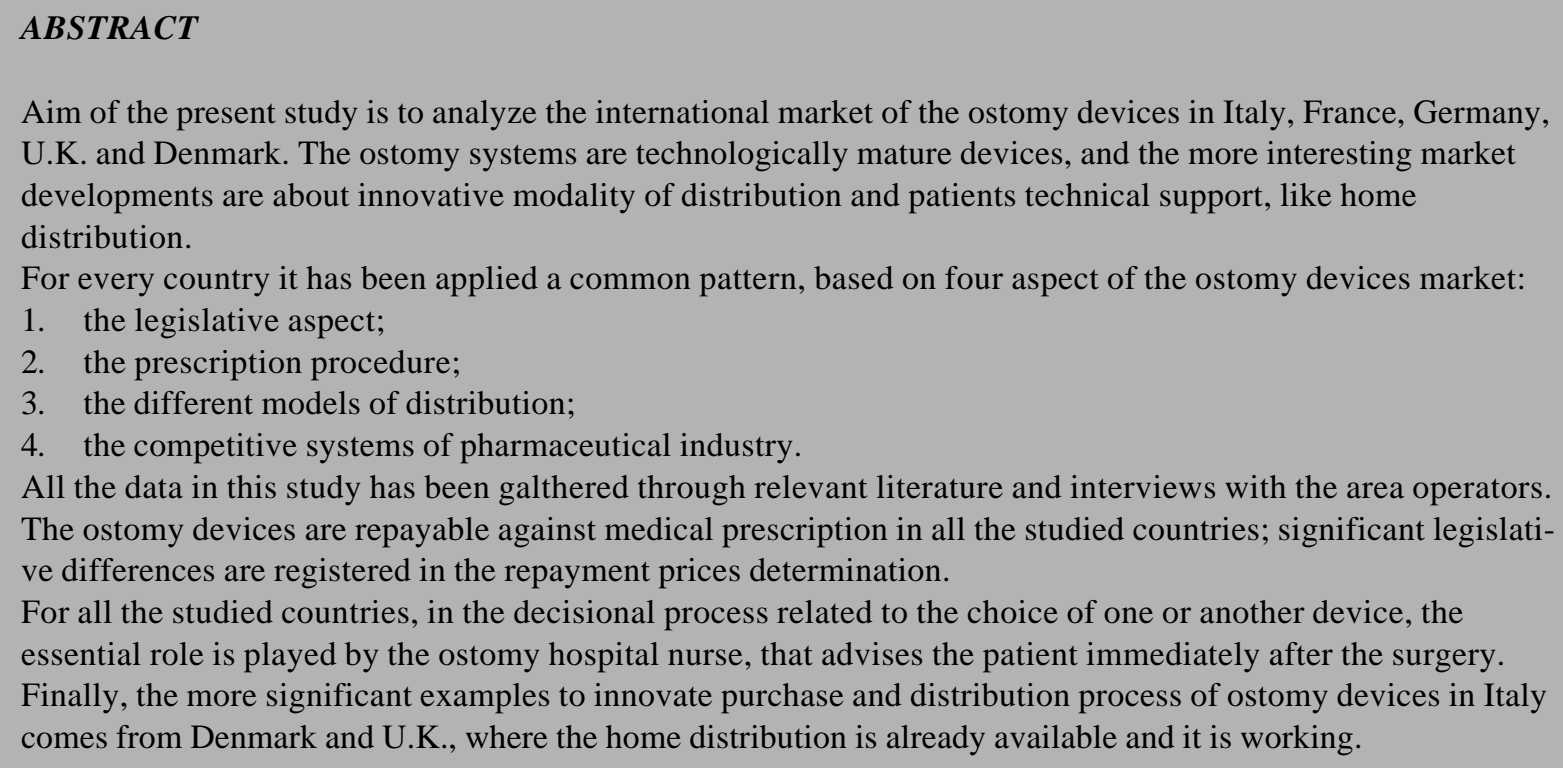

Aim of the present study is to analyze the international market of the ostomy devices in Italy, France, Germany, U.K. and Denmark. The ostomy systems are technologically mature devices, and the more interesting market developments are about innovative modality of distribution and patients technical support, like home distribution.

For every country it has been applied a common pattern, based on four aspect of the ostomy devices market:

1. the legislative aspect;

2. the prescription procedure;

3. the different models of distribution;

4. the competitive systems of pharmaceutical industry.

All the data in this study has been galthered through relevant literature and interviews with the area operators. The ostomy devices are repayable against medical prescription in all the studied countries; significant legislative differences are registered in the repayment prices determination.

For all the studied countries, in the decisional process related to the choice of one or another device, the essential role is played by the ostomy hospital nurse, that advises the patient immediately after the surgery. Finally, the more significant examples to innovate purchase and distribution process of ostomy devices in Italy comes from Denmark and U.K., where the home distribution is already available and it is working.

Farmeconomia e percorsi terapeutici 2000; 2 (2): 115-123

\section{INTRODUZIONE}

Il presente studio ha come obiettivo principale quello di analizzare a livello internazionale, in Italia e in altri quattro Paesi europei (Francia, Germania, Regno Unito e Danimarca), un segmento particolare del mercato sanitario: i dispositivi per stomia. Tali dispositivi sono prodotti tecnologicamente maturi, i cui sviluppi più interessanti di mercato appaiono collegati a modalità innovative di distribuzione e supporto tecnico ai pazienti, quali, ad esempio, la distribuzione domiciliare, in grado di soddisfare i bisogni dei pazienti in modo innovativo rispetto ai canali distributivi tradizionali.

La scelta delle quattro nazioni estere è motivata da diversi fattori: Francia, Germania e Regno Unito sono state incluse nell'analisi a causa della rilevanza territoriale e politica dei rispettivi mercati all'interno dell'Unione Europea, mentre per la Danimarca la scelta è stata motivata dalla diffusione capillare del sistema di assistenza domiciliare sul territorio nazionale (caratteristica probabilmente favorita dalla limitata estensione geografica e dalla morfologia pianeggiante del territorio).
Le considerazioni finali dello studio sono rivolte a possibili indicazioni per il Servizio Sanitario Nazionale italiano, alla luce di quanto riscontrato in fase di analisi comparativa.

\section{TECNOLOGIA}

I dispositivi per stomia sono sacche di raccolta di effluenti organici, applicate all'altezza dell'addome dei pazienti che hanno subito interventi chirurgici di colostomia (ad esempio, pazienti oncologici al colon o al retto), ileostomia (tipicamente pazienti con patologie intestinali infiammatorie croniche) e urostomia (ad esempio, pazienti oncologici alla vescica). L'intervento chirurgico definitivo consiste nella procedura di Miles, alternativa a quella temporanea di Hartmann che consente il ripristino delle funzionalità dell'intestino entro un massimo tre mesi. Con la procedura di Miles si crea un collegamento con l'esterno del corpo attraverso un abboccamento all'addome; le complicanze che possono insorgere in seguito a questa operazione sono quelle cosiddette di malconfezionamento". $\square C E S A V$

(Centro di Economia Sanitaria A. e A. Valenti) dell'Istituto perle Ricerche

Farmacologiche M. Negri

Corrispondenza a: Dott. Livio Garattini, clo Centro di Economia Sanitaria "Angelo e Angela Valenti" (CESAV) Villa Camozzi Via Camozzi, 3 24020 Ranica $(B G)$ E-Mail:

liviogarattini@tiscalinet.it 
Il sistema di raccolta degli effluenti può essere monopezzo o a due pezzi: il primo è costituito da una borsa semplice, con un adesivo idrocolloide ipoallergenico applicabile direttamente all'addome; il secondo è composto da una placca rigida o flessibile (collegabile alla sacca vera e propria con un adesivo o con una flangia) e da una borsa a fondo chiuso o aperto (con o senza flangia, con o senza filtro). Il sistema a un pezzo è di concezione meno recente, quello a due, invece, permette di mantenere aderente al corpo solamente la parte fissa, sganciando la sacca vera e propria, e contribuendo a provocare, di conseguenza, meno dermatiti (soprattutto nei soggetti più anziani). I sistemi monopezzo o a due pezzi sono fra loro alternativi.

Oltre a questa distinzione merceologica di carattere tecnico, ne esiste un'ulteriore collegata alla patologia specifica del paziente (colostomia, ileostomia o urostomia), Le sacche colon, di colore opaco, sono completamente chiuse e raccolgono gli effluenti solidi a livello del colon. Le sacche ileo (di colore opaco o trasparenti) raccolgono effluenti solido-liquidi a livello dell'ileo; dal momento che si accumulano rifiuti liquidi con una discreta frequenza, ad una estremità della sacca è posto un fermaglio per permettere lo svuotamento della stessa. Infine, le sacche uro (trasparenti) raccolgono effluenti liquidi dalla vescica e, considerata la maggior frequenza di immissione liquida, è possibile svuotarle di volta in volta tramite un apposito rubinetto posizionato sul fondo.

\section{MATERIALI E METODI}

L'analisi è stata condotta applicando uno schema comune in tutti i Paesi indagati (Italia, Francia, Germania, Gran Bretagna e Danimarca). Innanzitutto, viene fornita una panoramica degli aspetti normativi del settore sanitario oggetto di studio; successivamente, vengono esaminati l'iter prescrittivo e le modalità distributive dei dispositivi per stomia. Infine, l'analisi si conclude cercando di descrivere il mercato in termini di concorrenzialità e di segmentazione delle imprese. Le informazioni necessarie allo studio sono state raccolte mediante:

- analisi della letteratura nazionale ed internazionale;

- interviste condotte in ciascuna nazione con un gruppo di esperti scelti fra rappresentanti delle autorità sanitarie pubbliche, medici e dirigenti delle aziende del settore.

Sfortunatamente, la sensazione iniziale di difficoltà di raccolta di informazioni quantitative affidabili su un mercato di nicchia così specifico è stata confermata nel corso della ricerca. Infatti, la maggior parte delle informazioni espo- ste proviene dalle interviste effettuate agli operatori del settore.

\section{ITALIA}

Normativa di riferimento

La normativa riguardante gli ausili per stomizzati è riconducibile solamente a quanto riportato nell'allegato $1 \mathrm{del}$ Nomenclatore Tariffario (N.T.) in termini di quantitativo rimborsabile dal Servizio Sanitario Nazionale (SSN).

Da un'analisi condotta, è emerso come le modalità di determinazione dei prezzi di rimborso dei suddetti dispositivi varino da un'ASL all'altra, definendo uno scenario abbastanza eterogeneo. In assenza di un prezzo nazionale di riferimento, il ricorso a procedure pubbliche di acquisto è molto sporadico, mentre risulta molto più frequente il rimborso sulla base del prezzo indicato nel precedente N.T.

La fornitura rimborsabile di sacche, per colostomizzati e per ileostomizzati, è di 60 pezzi al mese, sia che si tratti del sistema monopezzo sia di quello a due pezzi; in questo secondo caso, va aggiunto un numero massimo di 10 placche (il primo materiale a contatto con la pelle). Diversamente, le borse per urostomia vengono rimborsate fino ad un massimo di 30 pezzi mensili, unitamente a 20 placche.

I sistemi monopezzo e a due pezzi riferiti agli ausili per colo/ileo/urostomizzati sono fra loro alternativi; pertanto, è ipotizzabile in questo caso un ordinativo congiunto per i due diversi tipi di ausili, per un quantitativo complessivo non superiore al tetto massimo previsto. Va precisato, tuttavia, che l'ammontare mensile di dispositivi a carico del SSN è da intendersi per ogni singola stomia, e non per ogni singolo paziente; quindi, nell'eventualità di persone affette da stomie differenti, il rimborso totale corrisponde alla somma dei quantitativi massimi previsti per le singole patologie.

\section{Iter prescrittivo}

Il problema primario per il paziente nella fase immediatamente successiva all'intervento chirurgico è quello di trovare il dispositivo che meglio risponda alle proprie esigenze personali, in termini di compatibilità con la propria conformazione fisica. Formalmente, il paziente dovrebbe aver la possibilità di provare tutte le diverse tipologie di sacca e, quindi, di effettuare la propria scelta in modo informato; in realtà, questa operazione, svolta in ambito ospedaliero, viene pilotata dall'intervento di una figura professionale esperta del settore che indirizza la scelta del paziente, proponendo presumibilmente quella che, fra le varie alternative a parità di efficacia, soddisfa il fornitore 
con cui intrattiene rapporti migliori. Questa figura può essere un medico, oppure, più frequentemente, un infermiere professionale o un enterostomista (cioè un infermiere in possesso di un titolo di formazione specifico). La fase post-chirurgica è, quindi, quella critica sotto il profilo commerciale, in cui l'azienda produttrice deve concentrare i propri sforzi promozionali per ottenere maggiori probabilità di fidelizzare un paziente al proprio prodotto. In effetti, la prassi ha dimostrato che il paziente è propenso a cambiare la scelta del prodotto solo nel periodo strettamente post-operatorio, mentre con il trascorrere del tempo difficilmente sarà spinto a modificare la propria scelta, a meno che non emerga un' evidente incompatibilità cutanea con il dispositivo in uso (evento, peraltro, abbastanza raro).

Gli enterostomisti in Italia sono attualmente circa 220 e si sono organizzati in un' associazione di categoria autocertificata, denominata AIOSS (Associazione Italiana Operatori Sanitari Stomaterapisti), mentre, sul fronte dell'utenza, i pazienti stomizzati hanno fondato una associazione denominata AISTOM (Associazione Italiana STOMizzati).

Trattandosi di prodotti sostanzialmente omogenei, il dispositivo fornito da un'azienda dovrebbe essere presentato come equivalente a quello della concorrenza (il servizio e il prezzo sono i veri fattori distintivi). Tuttavia, spesso il medico prescrittore (cioè lo specialista ospedaliero), nonostante sia tenuto per legge a riportare solo il codice del prodotto prescritto (senza indicarne la marca), scrive il nome commerciale del prodotto sulla ricetta, oppure consegna al paziente un foglietto di promemoria riportante il nome del fornitore stesso. La giustificazione logica di tale comportamento è quella di non indurre in confusione il paziente e non permettere al farmacista possibili cambiamenti di marca rispetto a quella già sperimentata dal paziente stesso.

Il ruolo del medico di famiglia consiste semplicemente nel certificare la prescrizione o nel prescrivere i rinnovi di ricetta, risultando, quindi, di natura prettamente burocratica e priva di discrezionalità. Le prescrizioni sono in genere rinnovabili mensilmente.

\section{Distribuzione}

La distribuzione dei dispositivi per stomia avviene attraverso canali differenti a seconda delle disposizioni regionali vigenti. Una prima possibilità, ormai poco utilizzata, è rappresentata dalla distribuzione diretta da parte delle ASL (ad esempio, parte della Lombardia, dell'Emilia Romagna, della Toscana, delle Marche e della Sicilia, Piemonte, Liguria, Abruzzo, Lazio e Basilicata); in questa ipotesi, l'onere della distribuzione agli aventi diritto è a carico dell' ASL. Attraverso l'impiego di risorse proprie (magazzino e personale), il servizio farmaceutico garantisce la disponibilità degli ausili ai pazienti, i quali si recano direttamente presso le strutture dell'ASL per ritirare la propria fornitura. Anche se l'ASL ottiene un risparmio monetario rispetto all'importo totale della fornitura, spesso vengono sottostimate le ingenti risorse aggiuntive conseguenti allo stoccaggio dei prodotti.

Una seconda possibilità, invero la più diffusa sul territorio nazionale, è costituita dalla distribuzione indiretta attraverso rivenditori convenzionati, tipicamente le farmacie e le sanitarie: in questo caso, l'ASL ricorre all'esterno per l'intera gestione del processo distributivo. Il vantaggio immediato è il trasferimento dell'onere dell'investimento al di fuori della propria struttura; di converso, la mancanza di strumenti di controllo sull'adeguatezza dei margini riconosciuti al sistema distributivo è il punto debole di questa scelta.

Una terza forma distributiva, ancora in fase embrionale, riguarda la distribuzione a domicilio: i prodotti vengono consegnati direttamente a casa dei pazienti, senza che questi ultimi debbano recarsi personalmente presso altre strutture. Questo progetto riassume concettualmente i vantaggi potenziali dei due sistemi precedentemente illustrati, permettendo un servizio personalizzato ai pazienti a costi contenuti. Infatti, il vantaggio per l'utente, scegliendo questa terza opzione, è una consegna domiciliare periodica che soddisfa le esigenze del singolo individuo in termini di comodità, programmazione e riservatezza (spesso i pazienti preferiscono tenere nascosto il proprio stato di salute); in aggiunta, viene garantito da parte del fornitore un servizio di consulenza, attraverso una linea verde e una riduzione delle pratiche burocratiche per il rinnovo della prescrizione. I vantaggi potenziali per le ASL sono: un risparmio in termini di spesa rispetto a quella dei canali distributivi esterni; una razionalizzazione dei consumi attraverso il controllo diretto on-line fornito dalle aziende produttrici; un monitoraggio continuo del bacino di utenza grazie a uno scambio informativo continuo con il personale sanitario dell' azienda.

Questo tipo di progetto interessa maggiormente le ASL che non prevedono alcun servizio domiciliare infermieristico specifico, considerato il notevole apporto che può derivare dall'offerta di personale specializzato da parte dell'azienda fornitrice; problematica può risultare, invece, l'integrazione con il personale enterostomista degli ospedali, geloso della propria professionalità e tendenzialmente ostile a interventi percepiti come ingerenze esterne.

Il risparmio economico è una variabile a cui sono più sensibili le ASL che consegnano que- 
sti prodotti con canali esterni, in particolare quelle che rimborsano alle farmacie e alle sanitarie in assenza di condizioni contrattuali particolari. Ad ostacolare questi tipi di progetti possono, invece, intervenire le associazioni delle farmacie, preoccupate dall'oggettivo timore di perdere clienti (al di là dell'esiguo fatturato in valori assoluti generato dai dispositivi per stomia).

\section{Analisi di mercato}

I pazienti stomizzati in Italia sono stimati in circa 40.000-45.000; il mercato domestico di dispositivi per stomia ammonta a circa $90 \mathrm{mi}-$ liardi di Lire è distribuito principalmente fra tre aziende: il leader ne detiene circa il $45 \%$, mentre i due rimanenti controllano una quota pari a circa il $20 \%$ ciascuna. Non esistono differenze sostanziali fra i prodotti di marche diverse: tutti devono riportare il marchio CE e la loro qualità deve rispecchiare specifici requisiti organolettici per poter essere inclusi, attraverso codici identificativi, fra le tipologie di prodotti del N.T.

\section{FRANCIA}

\section{Normativa di riferimento}

I dispositivi per stomia rientrano fra i prodotti interamente rimborsati ai pazienti e sono inclusi nel catalogo Tarif Interministériel des Prestations Sanitaires (TIPS); il prezzo riportato in questo catalogo si riferisce genericamente alla tipologia di sacca, in modo indifferenziato per tutti i prodotti delle marche concorrenti. Il prezzo di qualsiasi prodotto, una volta inserito nel catalogo TIPS, viene raramente aggiornato in termini tariffari, ragion per cui il modo migliore di ottenere aumenti di prezzo è quello di lanciare nuovi prodotti alternativi, riconosciuti come originali (a prescindere dall'effettivo tasso di innovazione) e, quindi, in grado di ottenere una nuova catalogazione.

La scelta del fornitore è tradizionalmente affidata al canale ospedaliero che, quindi, è utilizzato dalle aziende per fini prevalentemente promozionali; tuttavia, le sacche non vengono mai offerte in modo del tutto gratuito, sia per mantenere rapporti stabili con l'ufficio acquisti dell'ospedale, sia per mantenere l'opportunità formale di entrare in contatto diretto con i pazienti, stabilendo un rapporto di fiducia e garantendo loro un' assistenza tecnica. Le procedure di acquisto ospedaliere sono quelle tipiche del settore pubblico: gare o trattative private.

\section{Iter prescrittivo}

Le sacche per stomizzati sono prescritte sia dallo specialista ambulatoriale sia dal medico di famiglia, anche se entrambe le figure assumono in pratica il compito di semplici compilatori di ricette: il ruolo cruciale nella decisione d'acquisto è svolto solitamente dall'enterostomista o dall'infermiere specializzato ospedaliero, in quanto la scelta di legare un paziente a una determinata marca viene effettuata prevalentemente da queste figure professionali, subito dopo l'operazione. Gli enterostomisti in Francia sono circa 300 e svolgono la propria attività esclusivamente nei grandi ospedali, controllando circa il 30-40\% del mercato. Nel resto degli ospedali molte operazioni sono condotte in reparti privi di tale figura professionale (in realtà non riconosciuta come specializzazione ufficiale dalla normativa vigente); a fini promozionali, dunque, per le aziende produttrici diventa un target importante anche quello degli altri infermieri specializzati.

Riassumendo, il paziente è già stato indirizzato alla marca che dovrà richiedere quando ottiene la prescrizione del medico; sulla ricetta è comunque consentita anche l'indicazione specifica della marca della sacca prescritta. Va ricordato, infine, che la ricetta per la prescrizione di sacche per stomizzati viene rinnovata ogni tre mesi.

\section{Distribuzione}

Il paziente può ritirare il prodotto in farmacia utilizzando la prescrizione. Il farmacista è teoricamente libero di fissare un prezzo superiore a quello indicato dal TIPS all'atto della dispensazione, richiedendo la differenza al paziente; tuttavia, al farmacista non conviene normalmente questo tipo di atteggiamento, in quanto rischia di perdere il cliente (solitamente "consumatore pesante" anche di altri tipi di prodotti sanitari). La stessa ricetta rilasciata dal medico può essere utilizzata per il ritiro del prodotto anche presso le sanitarie (bandagistes).

In teoria, i dispositivi per stomia potrebbero essere venduti anche nei supermercati, ma, trattandosi di prodotti a basso rigiro e dal considerevole ingombro, questa categoria di esercizi non ha convenienza ad attrezzarsi sotto il profilo amministrativo per commercializzarli. A questo proposito, va ricordato che il farmacista solitamente non tiene in scorta i dispositivi per stomia, ma li ordina in giornata al grossista, venendo rifornito da quest'ultimo nel giro di poche ore.

\section{Analisi di mercato}

In Francia i pazienti aventi diritto sono circa 45.000 e il mercato domestico di dispositivi per stomia è stimato in 120 miliardi di Lire circa. Le aziende produttrici sono quelle presenti in tutti i principali paesi europei: il leader controlla circa il 35\% del mercato, mentre i due principali concorrenti si attestano su un valore pari a circa il 30\% ciascuno. Nessuna delle suddette 
aziende produttrici di dispositivi per stomia effettua distribuzione a domicilio, perché tale strategia trova tuttora un ostacolo insormontabile negli enterostomisti (timorosi di essere in qualche misura espropriati del paziente) e nei farmacisti (preoccupati delle ripercussioni in termini di fatturato).

\section{GERMANIA}

\section{Normativa di riferimento}

I dispositivi per stomia sono prodotti rimborsabili anche in Germania: le Krankenkassen coprono, infatti, i costi per l'acquisto di questi prodotti ai pazienti che presentano la ricetta medica. In mancanza di quest'ultima, gli utenti sono comunque abilitati a ritirare i prodotti per stomia, ma a proprie spese.

I dispositivi medici sono soggetti al sistema dei prezzi di riferimento nei Länder in cui tale sistema è in vigore. Di conseguenza, i prezzi variano da Länder a Länder e vengono fissati dalle associazioni delle Krankenkassen regionali (Spitzenverbande der Krankenkassen), senza alcuna cadenza periodica temporale prefissata per la revisione degli stessi. Èattualmente in discussione l'ipotesi di adottare un sistema di prezzi di riferimento più disaggregato in funzione delle diverse tipologie di prodotti. Qualora il prezzo di riferimento fosse inferiore a quello proposto al dettaglio, il farmacista dovrebbe spiegare all'utente il problema dell'eventuale costo aggiuntivo a carico di quest'ultimo; tuttavia, anche in Germania il farmacista opta spesso per dispensare comunque il prodotto al prezzo di riferimento, al fine di non rischiare di perdere il cliente (potenziale acquirente anche di altri prodotti sanitari).

Nei Länder in cui il sistema di prezzi di riferimento non è mai stato adottato o è stato $a b$ bandonato, si ricorre a negoziazioni dirette fra le Krankenkassen e le associazioni locali di farmacie, le sanitarie o i distributori stessi. È utile sottolineare che anche in Germania il prezzo di rimborso è differenziato per tipologia di sacca (a uno o due pezzi, aperte o chiuse) e risulta indistinto per tutti i produttori, indipendentemente dalla marca.

Il margine di retribuzione del grossista è liberamente negoziato con il produttore, mentre quello del farmacista si ricava come differenza fra il prezzo di riferimento e quello comprensivo del margine di intermediazione del distributore stesso, oppure, molto raramente, può scaturire da una negoziazione diretta fra produttore e farmacista stesso.

\section{Iter prescrittivo}

Anche in questo Paese il decisore di acquisto per i dispositivi per stomia è l'enterostomista ospedaliero, il quale indirizzail paziente nella scelta del prodotto successivamente all'intervento chirurgico. Esistono anche enterostomisti con Krankenkassen che seguono il paziente ambulatorialmente; tali figure hanno, però, un peso decisionale marginale nella scelta del prodotto, in quanto curano pazienti che sono già utilizzatori di sacche per stomia (la loro funzione è di assistenza e controllo).

Il paziente stomizzato riceve, all'atto della dimissione ospedaliera, un piccolo quantitativo di prodotti (il numero strettamente necessario per i primi giorni); successivamente, è tenuto a recarsi dal medico prescrittore, il quale si limita solitamente a confermare la prescrizione del tipo di dispositivo rilasciato all' atto della dimissione. Infine, esibendo tale prescrizione, il paziente può ottenere i prodotti gratuitamente presso tutte le farmacie e le sanitarie o direttamente a domicilio, in base agli accordi presi fra le Krankenkassen e i fornitori sul territorio di residenza del paziente stesso.

\section{Distribuzione}

In Germania le farmacie si avvalgono solitamente del "canale lungo" (così definito perché prevede un anello aggiuntivo, il distributore intermedio, nella catena distributiva) per la distribuzione di dispositivi per stomia; le sanitarie, invece, prediligono il canale "corto" (facilitato anche dall'esistenza di catene di sanitarie, in grado di far valere il proprio potere d'acquisto negoziando sconti consistenti direttamente con i produttori).

Il mercato tedesco dei prodotti per stomia risulta ripartito in quote quasi equivalenti, in termini di canali distributivi, fra le sanitarie (33\%), i distributori medicali (32\%), ossia i grossisti che eseguono la consegna a domicilio, e le farmacie $(31 \%)$; segue, infine, il canale ospedaliero con il $4 \%$ (il valore percentuale trascurabile di quest'ultimo è collegato al ruolo prettamente promozionale dello stesso anche in Germania).

\section{Analisi di mercato}

In Germania i pazienti aventi diritto a dispositivi per stomia sono complessivamente circa 100.000, e il mercato interno di dispositivi è stimato in circa 300 miliardi di Lire.

Il $90 \%$ del mercato tedesco di dispositivi per stomia è controllato da quattro aziende, con le due principali che detengono una quota pari a circa un terzo ciascuna. Tutte le suddette aziende operano ad ampio raggio, ovvero offrono un assortimento completo per tutti i tipi di stomia (colo, ileo e uro), producendo sacche a uno e due pezzi e garantendo un'accurata assistenza postoperatoria. Le aziende marginali concentrano la propria attenzione esclusivamente su una determinata nicchia produttiva. 


\section{REGNO UNITO}

Normativa di riferimento

I dispositivi per stomia non sono attualmente classificati dal National Health Service (NHS) come prodotti generici, in quanto la tecnologia dei prodotti concorrenti non viene ancora considerata del tutto equivalente. Ècomunque opinione diffusa che i dispositivi per stomia potranno diventare generici nei prossimi anni.

Per essere commercializzati, anche in Inghilterra i dispositivi devono presentare il marchio $\mathrm{CE}$; la registrazione è depositata presso l' Agenzia dei dispositivi del NHS. I dispositivi per stomia sono inclusi nella classe IX C (ossia quella che include i prodotti valutati di limitata pericolosità per i pazienti) dei prodotti della Drug Tariff List (DTL), la lista dei prodotti rimborsabili dal NHS attraverso il canale farmacia.

La negoziazione dei prezzi da parte del NHS avviene direttamente con l'azienda produttrice e si basa sostanzialmente sul prezzo medio dei prodotti similari presi come riferimento nella DTL, a prescindere dalla pretesa innovazione che caratterizzerebbe $\mathrm{i}$ dispositivi più recenti.

\section{Iter prescrittivo}

La figura che tradizionalmente influisce maggiormente sulla prescrizione è l'enterostomista (300 in tutto il Paese), analogamente a quanto avviene negli altri Paesi. Tuttavia, la tendenza in atto nel NHS è quella di investire di un ruolo più centrale $\mathrm{i}$ medici ospedalieri e, in ultima analisi, i direttori generali degli Hospital Trusts. Infatti, il rapporto Calman, mirato a riorganizzare i servizi oncologici, ha incentivato la creazione di sponsorships dirette da parte delle aziende ai reparti di oncologia (tipicamente offrendo di coprire i costi del personale infermieristico). Queste sponsorships, generalmente della durata variabile da tre a cinque anni, sono soggette a trattativa privata (per circa il $50 \%$ dei casi), oppure aggiudicate attraverso gare d'appalto e hanno come logico ritorno aziendale da parte dell'aggiudicatario quello di poter orientare la scelta dei nuovi pazienti esclusivamente verso la propria marca. La ricetta del medico di medicina generale non è solitamente generica, ma specifica: oltre al modello di sacca prescritta, viene indicata anche la marca del prodotto (decisa, peraltro, anche in questo caso, a livello ospedaliero nella fase post-chirurgica).

\section{Distribuzione}

In Gran Bretagna, i dispositivi per stomia possono essere distribuiti dalle farmacie o da distributori specializzati nella dispensazione di dispositivi a domicilio, denominati Dispensing
Appliance Contractors (DACs). Il canale tradizionale delle farmacie viene rimborsato con una fee for service fissa, diversamente dai DACs che percepiscono una percentuale proporzionale al numero di prescrizioni evase.

Tale ricarico, che tende chiaramente a remunerare maggiormente il distributore di dispositivi rispetto al canale farmacia, appare giustificato dal fatto che quest'ultima, rispetto ai DACs, può contare anche sulle entrate derivanti dalla vendita di una ben più ampia gamma di prodotti (ad esempio, i farmaci).

Può anche succedere che le catene di farmacie, per mantenere alta la fedeltà dei propri clienti, consegnino i prodotti per stomia a domicilio, pur non percependo alcuna fee per tale servizio.

\section{Analisi di mercato}

I pazienti stomizzati nel Regno Unito sono stimati complessivamente in 80.000-100.000 e il mercato domestico di dispositivi per stomia è stimato in 140 miliardi di Lire circa.

Dal punto di vista dei fornitori, anche il mercato inglese risulta sostanzialmente suddiviso fra quattro aziende che, complessivamente, controllano 1' $80 \%$ del totale. Tutti i produttori principali hanno deciso di organizzarsi anche come distributori a domicilio, adottando soluzioni sia interne sia in outsourcing.

\section{DANIMARCA}

\section{Normativa di riferimento}

I dispositivi per stomia sono classificati come dispositivi medicali e vengono regolati dalla legge danese sulla sicurezza sociale che garantisce pieno supporto alle persone colpite da malattie croniche. Di conseguenza, ogni prodotto per stomia viene fornito gratuitamente da parte del Servizio Sanitario Nazionale danese a tutti i pazienti aventi diritto.

I Kommuner acquistano solitamente i dispositivi per stomia attraverso i distributori, in base ai listini che i produttori forniscono ai distributori stessi. Alcune autorità sanitarie locali negoziano con i distributori prezzi più competitivi per la fornitura a domicilio dei pazienti, indipendentemente dalla marca dei prodotti consegnati. Saranno i grossisti a negoziare successivamente con i produttori il proprio margine, cercando di ottenere i dispositivi al prezzo più conveniente. Il rapporto commerciale fra produttore e Kommuner viene così traslato a "monte" della catena distributiva.

Nei Kommuner di maggiori dimensioni possono essere indette apposite gare, con l'obiettivo di selezionare il distributore che riesce a effettuare la distribuzione a domicilio a costi più contenuti, grazie allo sfruttamento delle 
possibili economie di scala ottenibili mediante l'inserimento nel contratto d'appalto anche di prodotti diversi (quali ad esempio, quelli per suture e piaghe).

Sotto il profilo commerciale, si può affermare che non è molto conveniente per le aziende produttrici discostarsi in misura eccessiva dai margini mediamente concessi ai distributori da parte dei concorrenti, visto che correrebbero il rischio di innescare un clima di tensione commerciale, con prezzi al ribasso e contraccolpi negativi per la redditività di tutti gli operatori di mercato. Gli effetti prevedibili di un'accresciuta concorrenzialità sui prezzi sarebbero quelli di incentivare i distributori a promuovere i prodotti dell' azienda che offre maggiori sconti, stimolando il cambio della marca da parte dei pazienti, invadendo l'area di azione degli infermieri ospedalieri (veri "influenzatori” della prima scelta anche in Danimarca) e minando, in ultima analisi, l'attività promozionale dei fornitori stessi. Infatti, anche il personale delle aziende distributrici potrebbe assumere una certa influenza sui pazienti, spesso molto anziani, soprattutto per i frequenti contatti diretti (e quindi le solide relazioni umane) che instaurano con questi ultimi.

Nessun Kommuner interviene direttamente nel servizio di consegna a domicilio, delegando sempre tale attività al distributore con cui stipula uno specifico contratto.

Il canale farmacia ha un ruolo trascurabile in questo mercato, dal momento che l'utente riceve i prodotti direttamente a domicilio tramite il distributore e, solo in caso di estrema necessità, si rivolge al dettagliante. I dispositivi distribuiti tramite farmacia sono rimborsati dal SSN danese, ma possono comportare un copagamento nel caso in cui i margini esigui spingano il farmacista a praticare un prezzo superiore al valore di rimborso fissato dai Kommuner.

\section{Iter prescrittivo}

L'iter prescrittivo e la dispensazione dei dispositivi ai pazienti stomizzati risultano molto precisi: in seguito a intervento chirurgico, il medico o l'infermiere ospedaliero compilano l'apposito modulo riportante le necessità del degente e firmano la richiesta di rimborso per il paziente. Successivamente, il modulo viene inviato al Kommuner per l'autorizzazione al rimborso delle sacche alla dimissione del paziente. Contestualmente, viene spedita una copia della richiesta al distributore con il quale è stato siglato l'accordo di consegna a domicilio.

Nel caso specifico dei pazienti stomizzati, le autorità locali non frappongono alcun ostacolo formale al rimborso: senza effettuare ulteriori controlli, infatti, forniscono al paziente un buo- no d'acquisto (naturaliebevilling) che copre tutti i prodotti di cui ha bisogno; ciò significa che al paziente verrà recapitato a domicilio tutto il materiale indicato sul buono d'acquisto stesso.

La fattura di pagamento viene poi spedita dal distributore direttamente ai Kommuner.

\section{Distribuzione}

Come già ampiamente sottolineato, i Kommuner stipulano contratti direttamente con le aziende private di distribuzione domiciliare specializzate nella consegna di dispositivi biomedicali.

Il distributore di dispositivi medici è responsabile della correttezza della consegna, da effettuare esclusivamente in base a quanto indicato nel buono d' acquisto in possesso del paziente.

I distributori forniscono, oltre ai pazienti a domicilio, anche le case di riposo. In questo secondo caso, peraltro, è ancora molto diffusa l'abitudine da parte di queste strutture lungodegenziali ad acquistare $i$ prodotti in modo indipendente, anche qualora il Kommuner abbia indetto una gara di fornitura, rendendo il potere d'acquisto del Servizio Sanitario Nazionale danese molto frammentato.

In teoria, potrebbero competere per la distribuzione a domicilio anche i grossisti farmaceutici, ma i loro margini sarebbero difficilmente competitivi, in quanto i mezzi da loro utilizzati e le prassi distributive sono troppo dispendiosi rispetto alle esigenze dei dispositivi per stomia (distribuiti con frequenze più basse $\mathrm{e}$ non richiedenti autoveicoli caratterizzati da requisiti specifici).

\section{Analisi di mercato.}

In Danimarca i pazienti aventi diritto a dispositivi per stomia sono complessivamente circa 2.000 e il mercato interno di dispositivi per stomia è stimato in circa 5 miliardi di Lire.

Dal punto di vista della produzione, il mercato è suddiviso fra quattro aziende: il leader copre il $50 \%$, le due principali concorrenti il 20 $\%$ ciascuna e la rimanente circa il $10 \%$.

\section{ANALISI COMPARATIVA}

In tutti i Paesi analizzati i dispositivi per stomia sono classificati come dispositivi medici e vengono integralmente rimborsati dietro presentazione di ricetta medica.

Differenze normative significative si registrano, invece, nella determinazione dei prezzi di rimborso. In Italia le modalità di definizione dei prezzi dei dispositivi per stomia variano anche in modo sostanziale da un'ASL all'altra: se da un lato è frequente il rimborso sulla base 
dei prezzi del N. T. non più in vigore, dall'altro il ricorso a procedure pubbliche di acquisto è molto raro. In Francia e nel Regno Unito i prezzi sono fissati a livello nazionale (nelle liste del TIPS e della DTL, rispettivamente). I prezzi in Germania variano da Länder a Länder e vengono fissati dalle associazioni delle Krankenkassen regionali. A differenza degli altri Paesi analizzati, in Danimarca non esiste alcuna negoziazione fra i Kommuner e i produttori di dispositivi per stomia. Tali prodotti rientrano nel capitolato d'affari fra il distributore domiciliare e i Kommuner stessi. Più precisamente, le autorità locali concordano con i distributori il prezzo della fornitura a domicilio dei pazienti, indipendentemente dalla marca dei prodotti consegnati; successivamente, sono i grossisti a negoziare direttamente con i produttori il proprio margine. La tendenza generale è quella di mantenere contemporaneamente $i$ rapporti di fornitura con più aziende, al fine di soddisfare le richieste di tutti i pazienti (a prescindere dalla marca cui sono "fidelizzati").

In tutti i Paesi analizzati il ruolo chiave nel processo decisionale relativo alla scelta della marca della sacca è ricoperto dall'enterostomista ospedaliero (o semplicemente dall' infermiere, qualora la prima figura non sia presente), il quale indirizza il paziente sul prodotto scelto successivamente all'intervento chirurgico. In Italia, Francia e Germania il medico prescrittore (lo specialista per la prima ricetta e il medico di famiglia per i rinnovi successivi), nonostante sia tenuto a riportare solo il codice del prodotto prescritto, spesso indica anche la marca del dispositivo; tuttavia, tale indicazione è di scarsa importanza, in quanto è comunque il paziente stesso a ricordare al medico il prodotto che sta già utilizzando. Il ruolo successivo del medico di famiglia risulta, quindi, di natura prettamente amministrativa e del tutto ininfluente rispetto alla scelta della marca. Nel Regno Unito, all'interno del NHS, si registra la tendenza a attribuire un ruolo più centrale ai managers dei Trusts, attraverso l'incentivazione di sponsorships dirette da parte delle aziende ai reparti di oncologia, finalizzate alla copertura dei costi del servizio infermieristico. In Danimarca l'iter prescrittivo per i pazienti stomizzati è molto standardizzato: in seguito all'intervento chirurgico, il medico (o l'infermiere ospedaliero) compila l'apposito modulo riportante le caratteristiche del paziente e firma la richiesta di rimborso. Successivamente, il modulo viene inviato al Kommuner per autorizzare il rimborso e una copia viene spedita al distributore con il quale è stato concluso l'accordo di consegna a domicilio.

In Italia esistono principalmente due sistemi di distribuzione dei dispositivi per stomia. Il primo (fenomeno esclusivamente italiano, peraltro in netta diminuzione) prevede la distribuzione diretta da parte dell'ASL, la quale si accolla l'onere della distribuzione ai soggetti aventi diritto. Infatti, attraverso l'impiego di risorse proprie (magazzini e personale), il servizio farmaceutico garantisce la consegna degli ausili ai pazienti, i quali si recano direttamente presso le strutture pubbliche per ritirare i prodotti. Il secondo sistema, di gran lunga prevalente, è la distribuzione indiretta attraverso rivenditori convenzionati (farmacie e sanitarie); in questo caso, l'ASL delega agli esercizi commerciali al dettaglio l'intera gestione del processo. Questa via di distribuzione è adottata anche in Francia, Germania e Regno Unito.

Una terza via distributiva, ancora in fase embrionale in Italia, ma già presente parzialmente in Germania e Regno Unito e a pieno regime in Danimarca, è quella della distribuzione a domicilio: i prodotti vengono consegnati direttamente a casa dei pazienti dai produttori o dai distributori.

Il servizio di distribuzione domiciliare riassume concettualmente i vantaggi potenziali dei due sistemi precedentemente illustrati, con possibili risparmi in termini economici rispetto alle tariffe di rimborso al farmacista e, soprattutto, la possibilità di monitorare più facilmente i consumi.

I principali produttori di dispositivi per stomia sono quattro e risultano presenti in tutti i Paesi analizzati. La quasi totalità del mercato è detenuta da queste aziende, anche se le quote di mercato dei singoli produttori variano molto da paese a paese. Tale concentrazione, probabilmente giustificata dall'esiguità dei singoli mercati nazionali, sembra indicare una certa affidabilità in termini di qualità dei prodotti in questo segmento, ma, d'altro canto, anche una probabile scarsa propensione alla concorrenza sui prezzi, tipica di un mercato di tipo oligopolistico.

\section{CONSIDERAZIONI FINALI}

Le caratteristiche principali dei dispositivi per stomia, riscontrati in tutti i Paesi analizzati, appaiono quelle di essere prodotti sostanzialmente a tecnologia matura (e, quindi, sostanzialmente equivalenti sotto il profilo produttivo), ma percepiti dal "consumatore" come insostituibili a livello di marca, principalmente a causa del "vissuto emotivo" riconducibile alla gravità della patologia di cui ha sofferto il paziente stesso; in quest'ottica, risulta cruciale il ruolo giocato dal personale sanitario (soprattutto quello infermieristico) nella scelta del primo prodotto utilizzato. Alla luce di queste ca- 
ratteristiche, la conseguenza logica sulla trasparenza di questo mercato è rappresentata dall'ambiguità dei rapporti che possono ingenerarsi fra infermieri ospedalieri e aziende produttrici, target principale degli sforzi promozionali di queste ultime. In tal senso, l'iniziativa inglese di incentivare sponsorships dirette da parte delle aziende ai reparti di oncologia, attraverso l'appalto del servizio infermieristico, sembrerebbe consentire di programmare la spesa indotta, da un lato, e, dall'altro, di orientare la scelta dei nuovi pazienti esclusivamente verso una certa marca, risolvendo "alla radice" l'ambigua discrezionalità concessa all'enterostomista.

Le caratteristiche di fidelizzazione del paziente al prodotto specifico, e della conseguente insostituibilità della sacca, differenziano in modo deciso questa tipologia di dispositivi da altre, quali, ad esempio i pannoloni, anche sotto il profilo dei rapporti con i "terzi paganti ". Infatti, mentre nel caso dei dispositivi per incontinenza una gara per la fornitura può avere classicamente un unico aggiudicatario, non rappresentando la fidelizzazione alla marca un ostacolo insormontabile, nel caso dei dispositivi per stomia appare evidente l'opportunità di condurre gare "multi-aggiudicatarie". In tal senso appaiono interessanti le modalità pubbliche di acquisto dei dispositivi adottate in Danimarca e nel Regno Unito che consentono di superare il problema della gara "mono-aggiudicataria": il ricorso ad accordi diretti fra distributori e autorità locali per la consegna a domicilio (a prescindere dalla marca consegnata) trasferisce sull'intermediario il problema della negoziazione dei prezzi dei singoli dispositivi con i diversi produttori.

Un'ulteriore conseguenza della fidelizzazione alla marca è che le quote di mercato delle aziende concorrenti subiscono nel breve periodo variazioni trascurabili, essendo le possibili azioni promozionali di successo limitate quasi esclusivamente ai nuovi pazienti; tale caratteristica connota il segmento dei dispositivi per stomia come mercato sostanzialmente stabile sotto il profilo della concorrenza.

In conclusione, dall'analisi comparativa è emerso come gli esempi più all'avanguardia da cui il SSN italiano può trarre indicazioni utili per innovare i processi di acquisto e la distribuzione dei dispositivi per stomia sembrano provenire dagli altri servizi sanitari pubblici esaminati, nella fattispecie quelli danese e inglese.

\section{BIBLIOGRAFIA}

1. Sanderson ER. Henri Hartmann and the Hartmann operation. Arch Surg 1980;115(6):792-3.

2. Corsi F. Italia. In Garattini L. (a cura di) I dispositivi medici nei principali paesi europei. 1999;27-46.

3. Tediosi F. Francia. In Garattini L. (a cura di) I dispositivi medici nei principali paesi europei. 1999;48-58.

4. Chiara G. Giuliani G. Garattini L. Il sistema dei prezzi di riferimento: il caso tedesco. Bollettino SIFO 1997;43 (2):48-55.

5. Garattini L. Tediosi F. L'ossigenoterapia domiciliare in cinque paesi europei: un'analisi comparativa. Mecosan 2000;35:137-148.

6. Department of Health. Drug Tariff List. London, May 2001.

7. The expert advisory group on cancer. A policy framework for commissioning cancer services. April 1995.

8. Black P. Practical stoma care. Nursing standard 2000;14(41):47-53.

9. Tediosi F. Garattini L. Il Servizio Sanitario Danese. ASI 1999; 38:6:9.

10. Cornago D. Garattini L. Analisi comparativa del mercato dei dispositivi per incontinenza in cinque paesi europei. Farmeconomia e percorsi terapeutici 2000;1(2):103-110. 